\title{
Mobility Improves Coverage of Sensor Networks
}

\author{
Benyuan Liu \\ Dept. of Computer Science \\ University of \\ Massachusetts-Lowell \\ Lowell, MA 01854
}

\author{
Peter Brass \\ Dept. of Computer Science \\ City College of New York \\ New York, NY 10031
}

\author{
Olivier Dousse \\ School of Computer and \\ Communication Sciences \\ EPFL \\ Lausanne, Switzerland
}

\author{
Philippe Nain \\ INRIA \\ 06902 Sophia Antipolis \\ France
}

\author{
Don Towsley \\ Dept. of Computer Science \\ University of Massachusetts \\ Amherst, MA 01002
}

\begin{abstract}
Previous work on the coverage of mobile sensor networks focuses on algorithms to reposition sensors in order to achieve a static configuration with an enlarged covered area. In this paper, we study the dynamic aspects of the coverage of a mobile sensor network that depend on the process of sensor movement. As time goes by, a position is more likely to be covered; targets that might never be detected in a stationary sensor network can now be detected by moving sensors. We characterize the area coverage at specific time instants and during time intervals, as well as the time it takes to detect a randomly located stationary target. Our results show that sensor mobility can be exploited to compensate for the lack of sensors and improve network coverage. For mobile targets, we take a game theoretic approach and derive optimal mobility strategies for sensors and targets from their own perspectives.
\end{abstract}

\section{Categories and Subject Descriptors}

C.2.1 [Computer-Communication Networks]: Network Architecure and Design-Network Communications, Wireless Communication.

\section{General Terms}

Design, Performance.

\section{Keywords}

Coverage, Sensor Network, Mobility.

Permission to make digital or hard copies of all or part of this work for personal or classroom use is granted without fee provided that copies are not made or distributed for profit or commercial advantage and that copies bear this notice and the full citation on the first page. To copy otherwise, to republish, to post on servers or to redistribute to lists, requires prior specific permission and/or a fee.

MobiHoc'05, May 25-27, 2005, Urbana-Champaign, Illinois, USA.

Copyright 2005 ACM 1-59593-004-3/05/0005 ...\$5.00.

\section{INTRODUCTION}

Recently, there has been substantial research in the area of sensor network coverage. The coverage of a sensor network represents the quality of surveillance that the network can provide, for example, how well a region of interest is monitored by sensors, and how effectively a sensor network can detect intruders (targets). It is important to understand how the coverage of a sensor network depends on various network parameters in order to better design and use sensor networks for different application scenarios.

In many applications, sensors are not mobile; they remain stationary after their initial deployment. The coverage of such a stationary sensor network is determined by the initial network configuration. Once the deployment strategy and sensing characteristics of the sensors are known, the network coverage can be computed and remains unchanged over time. Recently, there has been a strong desire to deploy sensors mounted on mobile platforms such as mobile robots. Such mobile sensor networks are extremely valuable in situations where traditional deployment mechanisms fail or are not suitable, for example, a hostile environment where sensors cannot be manually deployed or air-dropped. Also, in application scenarios such as atmosphere and ocean environment monitoring, sensors move with the surrounding air or ocean currents. The coverage of a mobile sensor network now depends not only on the initial network configurations, but also on the mobility behavior of the sensors.

While the coverage of a sensor network with immobile sensors has been extensively explored and is relatively well understood $[12,8,9,3,7]$, reseachers have only recently started to study the coverage of mobile sensor networks. Most of this work focuses on algorithms to reposition sensors in desired positions in order to enhance network coverage $[5,1,10,15,14]$. More specifically, these proposed algorithms strive to spread sensors in the field so as to maximize the covered area. The main differences among these works are how exactly the desired positions of sensors are computed. Although the algorithms can adapt to changing environments and recompute the sensor locations accordingly, sensor mobility is exploited essentially to obtain a new stationary configuration that improves coverage after the sensors move to their desired locations.

In this paper, we study the coverage of a mobile sensor 
network from a different perspective. Instead of trying to achieve an improved network configuration as the end result of sensor movement, we identify and characterize the dynamic aspects of network coverage that depend on the movement of sensors.

Specifically, we are interested in the coverage resulting from the continuous movement of sensors. This coverage is not available if the sensors stop moving. We now briefly describe the coverage provided by the sensor movement, and the related research problems.

First, previously uncovered areas become covered as sensors move through them and covered areas become uncovered as sensors move away. As a result, the locations covered by sensors change over time, and a greater area will be covered over time than in the case where sensors are stationary. Also, a location is now not always covered. It alternates between being covered and not being covered. This raised the following question: what is the area coverage at a given time instant? what is the area coverage over a time interval? what are the durations of time that a location is covered and not covered?

Second, note that an initially undetected intruder will never be detected in a stationary sensor network if the intruder remains stationary or moves along an uncovered path. In a mobile sensor network, an intruder is more likely to be detected as the moving sensors patrol the field. Thus, sensor mobility provides a time-varying coverage not available in a sensor network with stationary sensors. This can significantly improve the intrusion detection capability of a sensor network. This raises the additional questions: how quickly can the sensors detect an intruder? how does the detection time depend on the searching strategy of the sensors? and what are the optimal mobility strategies for the sensors and the intruder?

The main contributions of our work are:

First, we characterize the fraction of the area covered by sensors for a randomly-deployed stationary sensor network. This characterization shows how the covered area depends on the density and sensing characteristics of the sensors. It provides a baseline for comparison with a mobile sensor network.

We then consider a random mobility model for sensors and study the effect of sensor mobility on various aspects of network coverage. We show that, while the fraction of the covered area at any given time instant remains unchanged, the area being covered during a time interval is improved as sensors move around. As a result, intruders and events that will never be detected in a stationary sensor network can now be detected in a mobile sensor network. This scenario is of great importance for applications that do not require simultaneous coverage of all locations at specific time instants.

Unlike a stationary sensor network where a location always remains either covered or not covered, due to sensor movement, a location is now only covered part of the time, alternating between being covered and not being covered. We characterize this coverage and covering time tradeoff by the fraction of time a location is covered, which is determined by the density and sensing range of the sensors, and does not depend on the sensor mobility. While this time average characterization shows, to a certain extent, how well a point is covered, it does not reveal the duration of the time that a point is covered and uncovered. The time scales of such durations are very important for network planning; they present the time granularity of the intrusion detection capability that a mobile sensor network can provide. To this end, we further characterize the time durations that a point is covered and not covered.

In some applications, it is important to detect intruders in the field of interest as quickly as possible. To this end, we study the detection time of an intruder, which is defined to be the time elapsed before the intruder is first detected. Intruders that will never be detected in a stationary sensor network can now be detected by moving sensors. We obtain the distribution of the detection time for a randomly located stationary intruder. The results suggest that sensor mobility can be exploited to effectively reduce the detection time of a stationary intruder when the number of sensors is limited.

For mobile intruders, the detection time depends on both the sensor and intruder mobility strategies. We take a game theoretic approach and study the best worst-case performance of a mobile sensor network in terms of the intruder detection time. For a given sensor mobility behavior, we assume that an intruder can choose its mobility strategy so as to maximize its detection time (its lifetime before being detected). On the other hand, sensors choose a mobility strategy that minimizes the maximum detection time resulting from the intruder's mobility strategy. We prove that the optimal sensor mobility strategy is for each sensor to choose its direction uniformly at random. The corresponding intruder mobility strategy is to remain stationary in order to maximize the time before it is detected.

The remainder of the paper is structured as follows. The network model and coverage measures are presented in Section 2. In Section 3, we study the fraction of the area being covered at specific time instants and during a time interval. In Section 4, we study the detection time for both stationary and mobile intruders. In Section 5, we review related work on the coverage of sensor networks. Finally, we summarize the paper in Section 6 .

Throughout the paper, shorthand $X \sim \exp (\mu)$ will stand for $P(X<x)=1-\exp (-\mu x)$, namely, the random variable $X$ is exponentially distributed with parameter $\mu$. Also, we will use the words intruder and target interchangeably.

\section{NETWORK AND MOBILITY MODEL}

In this section, we describe the network and mobility model used in this study, and define three coverage measures of a mobile sensor network.

\subsection{Sensing Model}

We assume that each sensor has a sensing radius, $r$. A sensor can only sense the environment and detect events within its sensing area, which is the disk of radius $r$ centered at the sensor. A point is said to be covered by a sensor if it is located in the sensing area of the sensor. The sensor network is thus partitioned into two regions, the covered region, which is the region covered by at least one sensor, and the uncovered region, which is the complement of the covered region. An intruder is said to be detected if it lies within the covered region.

\subsection{Coverage measures}

To study the coverage of a sensor network, we define the following three coverage measures. 
Definition 1. Area coverage: The area coverage of a sensor network at time $t, f_{a}(t)$, is the fraction of the geographical area covered by one or more sensors at time $t$.

Definition 2. Area coverage over a time interval: The area coverage of a sensor network during time interval $[0, t), f_{i}(t)$, is the fraction of the geographical area covered by at least one sensor at some point of time within $[0, t)$.

Definition 3. Detection time: Consider a target located at a random position outside of the covered area of a sensor network at time $t=0$. The detection time of the target, $X$, is defined to be the time at which the target first enters the sensing area of a sensor, i.e., the target is first detected by the sensor.

All three coverage measures depend not only on static properties of the sensor network (sensor density and sensing range), but also on the sensor mobility behavior. The characterization of area coverage at specific time instants is important for applications that require simultaneous coverage of the network, while the area coverage over a time interval is appropriate for applications that do not require simultaneous coverage of all locations at specific time instants, but rather prefer to cover the network within some time interval. The detection time measures how quickly a sensor network can detect a randomly located target that is not initially covered.

\subsection{Location and Mobility Model}

We consider a network consisting of a large number of sensors placed in a vast two-dimensional geographical region. For the initial configuration, we assume that, at time $t=0$, the locations of these sensors are uniformly and independently distributed in the region. Such a random initial deployment is desirable in scenarios where prior knowledge of the region of interest is not available. Also, random deployment can be the result of certain deployment strategies. For example, sensors may be air-dropped or launched via artillery in battlefields or unfriendly environments. Under this assumption, the sensor locations can be modeled by a stationary two-dimensional Poisson point process. Denote the density of the underlying Poisson point process as $\lambda$. The number of sensors located in a region $R, N(R)$, follows a Poisson distribution of parameter $\lambda\|R\|$, where $\|R\|$ represents the area of the region.

$$
P(N(R)=k)=\frac{e^{-\lambda\|R\|}(\lambda\|R\|)^{k}}{k !} .
$$

Since each sensor covers a disk of radius $r$, the initial configuration of the sensor network can be described by a Poisson Boolean model $B(\lambda, r)$. In a stationary sensor network, sensors do not move after being deployed and network coverage remains the same as that of the initial configuration. In a mobile sensor network, depending on the mobile platform and application scenario, sensors can choose from a wide variety of mobility strategies, from passive movement to highly coordinated and complicated motion. Sensors deployed in the air or ocean move passively according to external forces such as air or ocean currents; simple robots may have a limited set of mobility patterns, and advanced robots can navigate in a more complicated fashion.

In this work, we consider the following simple sensor mobility model. We assume sensors move independently of each other and with coordination among them. The movement of a sensor is characterized by its speed and direction. A sensor randomly chooses a direction $\theta \in[0,2 \pi)$ according to some distribution with probability density function $f_{\Theta}^{s}(\theta)$. The speed of the sensor, $V_{s}$, is randomly chosen from a finite range $v_{s} \in\left[0, V_{s}^{\max }\right]$, according to a distribution density function of $f_{V}^{s}(v)$.

Throughout the rest of this paper, we will refer to the initial sensor network configuration as a random sensor network $B(\lambda, r)$, and the above mobility model as random mobility model. The coverage measures defined above are functions of the actual locations of sensors, which vary for different realizations. In this work, we will study the expected value of the coverage measures.

\section{AREA COVERAGE}

In this section, we study and compare the area coverages of both stationary and mobile sensor networks. We analytically characterize the area coverage. We then discuss the implications of our results on network planning and show that sensor mobility can be exploited to compensate for the lack of sensors to increase the area being covered during a time period. Finally, we point out, due to the sensor mobility, a point is only covered part of the time; we further characterize this effect by determining the fraction of time that a point is covered.

Theorem 1. At any given time instant $t>0$, the area coverage of a stationary sensor network $B(\lambda, r)$ is

$$
f_{a}(t)=1-e^{-\lambda \pi r^{2}} .
$$

Proof. This is a result from stochastic geometry [4]. Here we present the key arguments of the proof.

Consider a bounded region $R$; the vacancy $V$ within $R$ is defined to be the area in $R$ not covered by sensors.

$$
V=\int_{R} \chi(x) d x
$$

where

$$
\chi(x)= \begin{cases}1 & x \text { is not covered } \\ 0 & \text { otherwise }\end{cases}
$$

Using Fubini's theorem we have

$$
E(V)=\int_{R} E\{\chi(x)\} d x .
$$

Consider an arbitrary point $x$ in region $R$ and denote the number of sensors which cover the point as $N$. Point $x$ is covered by sensors located within distance $r$. It follows immediately from the Poisson point process assumption that $N$ has a Poisson distribution with parameter $\lambda \pi r^{2}$. Therefore, we have

$E\{\chi(x)\}=P(x$ is not covered $)=P(N=0)=e^{-\lambda \pi r^{2}}$.

and

$$
E(V)=\int_{R} E\{\chi(x)\} d x=\|R\| e^{-\lambda \pi r^{2}} .
$$

Note that the above derivation is independent of $R$. The area coverage can thus be obtained as follows.

$$
f_{a}=1-\frac{E(V)}{\|R\|}=1-e^{-\lambda \pi r^{2}} .
$$




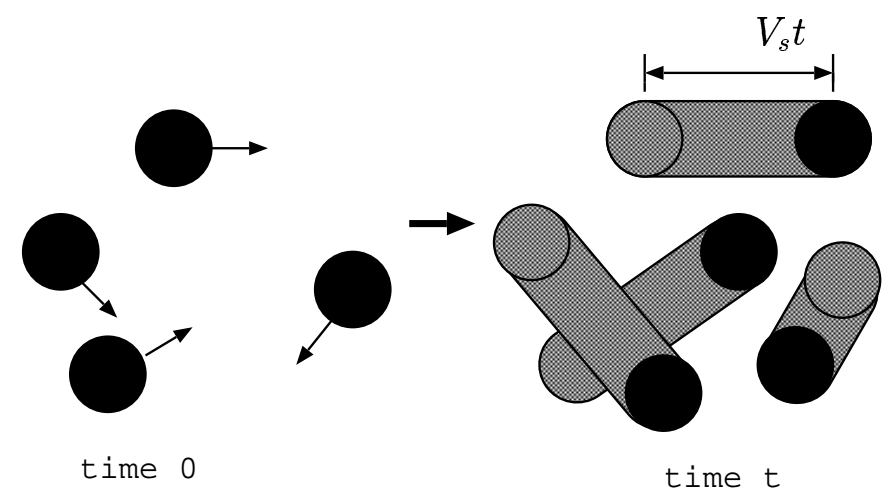

Figure 1: Coverage of mobile sensor network: the left figure depicts the initial network configuration at time 0 and the right figure illustrates the effect of sensor mobility during time interval $[0, t)$. The solid disks constitutes the area being covered at the given time instant, and the union of the shaded region and the solid disks represents the area being covered during the time interval.

This formula characterizes the dependence of area coverage on the network properties. It allows us to compute the fraction of the area being covered for a given sensor density and sensing range. For example, in order to achieve a desired area coverage $f_{a}\left(0<f_{a}<1\right)$ almost surely, the density required is given by

$$
\lambda=-\ln \left(1-f_{a}\right) / \pi r^{2} .
$$

In a stationary sensor network, a location always remains either covered or not covered. The area coverage does not change over time. The following theorem characterizes the effect of sensor mobility on network coverage.

THEOREM 2. Consider a sensor network $B(\lambda, r)$ at time $t=0$, with sensors moving according to the random mobility model.

1. At any time instant $t$, the fraction of area being covered

$$
f_{a}(t)=1-e^{-\lambda \pi r^{2}}, \quad \forall t \geq 0 .
$$

2. The fraction of area that has been covered at least once during time interval $[0, t)$ is

$$
f_{i}(t)=1-e^{-\lambda\left(\pi r^{2}+2 r E\left[V_{s}\right] t\right)} .
$$

3. The fraction of the time a point is covered is

$$
f_{t}=1-e^{-\lambda \pi r^{2}} \text {. }
$$

Proof. Given the initial node placement and the random mobility model, at any time instant $t$, the locations of the sensors still form a two dimensional Poisson point process of the same density [11, Theorem 9.14]. Therefore, the fraction of the area covered at time $t$ remains the same as in the initial configuration, $f_{a}(t)=1-e^{-\lambda \pi r^{2}}$.
As illustrated in Figure 1, during time interval $[0, t)$, each sensor covers a shape of a racetrack whose expected area is

$$
\alpha=E\left[\pi r^{2}+2 r V_{s} t\right]=\pi r^{2}+2 r E\left[V_{s}\right] t .
$$

where $E\left[V_{s}\right]=\int_{0}^{V_{s}^{\max }} f_{V}^{s}(V) d V$ represents the expected sensor speed.

As pointed out in [4], area coverage depends on the distribution of the random shapes only through its expected area. Therefore, we have

$$
f_{i}(t)=1-e^{-\alpha \lambda}=1-e^{-\lambda\left(\pi r^{2}+2 r E\left[V_{s}\right] t\right)} .
$$

While an uncovered location will be covered when a sensor moves within distance $r$ of the location, a covered location becomes uncovered as sensors covering it move away. As a result, a location is only covered part of the time. More specifically, a location alternates between being covered and not being covered, which can be modeled as an alternating renewal process. We use the fraction of time that a location is covered to measure this effect. The fraction of time that a location is covered equals the probability that it is covered at any given time instant, $f_{t}=1-e^{-\lambda \pi r^{2}}$. In the next section, we further characterize the time durations of a point being covered and not being covered.

At any specific time instant, the fraction of the area being covered in a mobile sensor network model described above is the same as in a stationary sensor network. This is because at any time instant, the positions of the sensor are still described by a Poisson Boolean model with the same parameters as in the initial configuration. Unlike in a stationary sensor network, areas initially not covered can now be covered as sensors move around in a mobile sensor network. Consequently, targets in the initially uncovered areas can be detected by the moving sensors.

Figure 1 illustrates the effect of sensor mobility on area coverage. The union of the solid disks constitutes the area coverage at given time instants. The area that has ever been covered during time interval $[0, t)$ is depicted as the union of the shaded region and the solid disks, occupying a larger portion of the total area.

Due to sensor mobility, the fraction of the area that has ever been covered increases and approaches one as time goes to infinity. The rate at which the covered area increases over time depends on the expected speed of sensor mobility. The faster sensors move, the more quickly the area is covered. Therefore, sensor mobility can be exploited to compensate for the lack of sensors to improve the area coverage over an interval of time. Note that the area coverage during a time interval does not depend on the distribution of the sensors' movement directions. Based on (4), we can compute the expected sensor speed required to ensure that a certain fraction of the area $\left(f_{0}\right)$ is covered within a time interval of length $t_{0}$.

$$
E\left[V_{s}\right]=-\frac{\lambda \pi r^{2}+\log \left(1-f_{0}\right)}{t_{0}}, \text { for } f_{0} \geq 1-e^{\lambda \pi r^{2}} .
$$

In a stationary sensor network, a location is either always covered or not covered, as determined by its initial configuration. In a mobile sensor network, as a result of sensor mobility, a location is only covered part of the time, alternating between being covered and not being covered. The fraction of time that a location is covered corresponds to the probability that it is covered, as shown in (5). Note 
that this probability is determined by the static properties of the network configuration (density and sensing range of the sensors), and does not depend on sensor mobility. This coverage-delay tradeoff can be exploited by applications that do not require simultaneous coverage of all locations at specific time instants.

\section{DETECTION TIME}

In the previous section, we characterized the fraction of area being covered at any given time and over a time interval. However, these measures do not reveal how quickly mobile sensors detect targets in the field. The time it takes to detect an intruding target is of great importance in many security-related applications. In this section, we study the detection time for both stationary and mobile targets. For mobile targets, we take a game theoretic approach and explore the best worst-case performance of the sensor network. We will derive optimal mobility strategies for targets and sensors that maximize or minimize the detection time.

To facilitate analysis and illustrate the effect of sensor mobility on the detection time, we make the assumption that all sensors move at a constant speed $v_{s}$. More general speed distributions can be approximated using the fixed speed scenario.

\subsection{Stationary Target}

We first consider the scenario where targets are stationary and do not initially fall into the coverage area of any sensor. Obviously, a stationary sensor network will never detect these targets; the detection times of these targets are infinite.

However, in a mobile sensor network, a target can be detected by any sensor passing within distance $r$ of it, where $r$ is the common sensing range of the sensors. The detection time of a stationary target characterizes how quickly the sensors can detect a randomly located target previously not detected.

ThEOREM 3. Consider a sensor network $B(\lambda, r)$ at time $t=0$, with sensors moving according to the random mobility model at a fixed speed $v_{s}$. Let $X$ be the detection time of a randomly located stationary target, we have

$$
X \sim \exp \left(2 \lambda r v_{s}\right)
$$

Proof. We divide the space evenly around point $p$ in $k$ directions $(k \rightarrow \infty)$. We now have $k$ sensor classes and sensors of class $i$ move in the direction $\theta_{i}=\frac{2 \pi i}{k}$. Since each sensor independently chooses its moving direction according to the same distribution, each sensor class is a thinning of the original point process. Therefore, sensor class $i$ forms a Poisson point process with density $\lambda_{i}=\lambda f_{\Theta}^{s}\left(\theta_{i}\right) \Delta \theta$, where $\Delta \theta=2 \pi / k$.

Point $p$ will be detected when a sensor moves within distance $r$ from the point. Now let $X_{i}$ (first hit time) be the time that point $p$ is first detected by a sensor of class $i$, the detection time $X$ is the minimum of all the first hit times, $X=\min X_{i}$.

Since all sensors of class $i$ move in the same direction $\theta_{i}$ at the same speed $v_{s}$, it is more convenient to consider the framework where sensors are relatively stationary and the target moves in the opposite direction $\left(2 \pi-\theta_{i}\right)$ at the sensor's speed $v_{s}$. The distance from point $p$ to the perimeter of the first sensor to contact it in direction $\left(2 \pi-\theta_{i}\right)$ is called the linear contact distance of that particular direction, and we denote it as $Y_{i}$. From [13, page 80], we know that $Y_{i}$ follows an exponential distribution with parameter $2 \lambda_{i} r$, i.e., $Y_{i} \sim \exp \left(2 \lambda_{i} r\right)$

Since $X_{i}=Y_{i} / v_{s}$, the first hit time in direction $i$ follows an exponential distribution with parameter $2 \lambda_{i} r v_{s}$,

$$
X_{i} \sim \exp \left(2 \lambda_{i} r v_{s}\right)
$$

Now the minimum of these exponential distributed first hit times is again an exponential distribution, with a parameter equal to the sum of the parameters of the individual exponential distributions:

$$
\begin{aligned}
\lim _{k \rightarrow \infty} \sum_{i=1}^{k} 2 \lambda_{i} r v_{s} & =\lim _{k \rightarrow \infty} \sum_{i=1}^{k} 2 \lambda f_{\Theta}^{s}\left(\theta_{i}\right) \Delta \theta r v_{s} \\
& =2 \lambda r v_{s} \int_{0}^{2 \pi} f_{\Theta}^{s}(\theta) d \theta \\
& =2 \lambda r v_{s} .
\end{aligned}
$$

Thus, we have $X \sim \exp \left(2 \lambda r v_{s}\right)$.

Compared to the case of stationary sensors where an undetected target always remains undetected, the probability that the target is not detected in a mobile sensor network decreases exponentially over time,

$$
P(X \geq t)=e^{-2 \lambda r v_{s} t}
$$

The expected detection time of a randomly located target is $E[X]=\frac{1}{2 \lambda r v_{s}}$, which is inversely proportional to the density of the sensors $(\lambda)$, the sensing range of each sensor $(r)$, and the speed of the sensor movement $\left(v_{s}\right)$. Note that the expected target detection time is independent of the sensor movement direction distribution density function, $f_{\Theta}(\theta)$. Therefore, in order to quickly detect a stationary target, one can add more sensors, use sensors with larger sensing ranges, or increase the speed of the mobile sensors.

Assuming there is a requirement that the expected time to detect a randomly located stationary target be smaller than a specific value $T_{0}$, we have

$$
\frac{1}{2 \lambda r v_{s}} \leq T_{0}
$$

or equivalently,

$$
\lambda v_{s} \geq \frac{1}{2 r T_{0}}
$$

Assuming the sensing range of each sensor is fixed, the above formula presents the tradeoff between sensor density and sensor mobility to ensure certain target detection time requirement. The product of the sensor density and sensor speed should be larger than a constant. Therefore, sensor mobility can be exploited to compensate for the lack of sensors, and vice versa.

In Theorem 2, we pointed out that a location alternates between being covered and not being covered, and then derived the fraction of time that a point is covered. While the time average characterization shows, to a certain extent, how well a point is covered, it does not reveal the duration of the time that a point is covered and uncovered. The time scales of such time durations are also very important for network planning; they present the time granularity of the intrusion detection capability that a mobile sensor network can provide. Having Theorem 3, we now characterize 
the time durations of a point being covered and not being covered.

Corollary 1. Consider a random sensor network $B(\lambda, r)$ at time $t=0$, with sensors moving according to the random mobility model. A point alternates between being covered and not being covered. Denote the time duration that a point is covered as $T_{c}$, and the time duration that a point is not covered as $T_{n}$, we have

$$
\begin{aligned}
T_{n} & \sim \exp \left(2 \lambda r v_{s}\right) \\
E\left[T_{c}\right] & =\frac{e^{\lambda \pi r^{2}}-1}{2 \lambda r v_{s}} .
\end{aligned}
$$

Proof. In the proof of Theorem 3, we have obtained the distribution of time for a randomly located point before it is first covered by a sensor. This time is the same as the time that a point is not covered. Therefore, we have $T_{n} \sim \exp \left(2 \lambda r v_{s}\right)$.

Since a point alternates between being covered and not being covered, the fraction of time a point is covered is

$$
f_{t}=\frac{E\left[T_{c}\right]}{E\left[T_{c}\right]+E\left[T_{n}\right]}=1-e^{-\lambda \pi r^{2}} .
$$

The last equality in the above equation is given in (5). Solving for $E\left[T_{c}\right]$, we obtain (8).

Let $T$ denote the period of a point being covered and not being covered, i.e., $T=T_{c}+T_{n}$. The expected value of the period is

$$
E[T]=E\left[T_{c}\right]+E\left[T_{n}\right]=e^{\lambda \pi r^{2}} / 2 \lambda r v_{s}
$$

\subsection{Mobile Target}

We now consider the case where a target is also mobile. The detection time of a mobile target depends not only on the mobility behavior of the sensors but also on the movement of the target itself.

Targets can adopt a wide variety of movement patterns. In this work, we will not consider specific target movement patterns. Rather, we approach the problem from a game theoretic standpoint and determine the optimal mobility strategies of the target and sensors.

Given the mobility model of the sensors, $f_{\Theta}^{s}(\theta)$, a target chooses the mobility strategy that maximizes its expected detection time. More specifically, a target chooses its speed $v_{t} \in\left[0, v_{t}^{\max }\right)$ and direction $\theta_{t} \in[0,2 \pi)$ so as to maximize the expected detection time. The expected detection time is a function of the sensor direction distribution density, target speed, and target moving direction. Denote the resulting expected detection time as $\max _{v_{t}, \theta_{t}} E\left[X\left(f_{\Theta}^{s}(\theta), \theta_{t}, v_{t}\right)\right]$; the sensors then choose the mobility strategy (over all possible direction distributions) that minimizes the maximum expected detection time. We have the following minimax optimization problem:

$$
\min _{f_{\Theta}^{s}(\theta)} \max _{\theta_{t}, v_{t}} E\left[X\left(f_{\Theta}^{s}(\theta), \theta_{t}, v_{t}\right)\right] .
$$

In the above problem statement, each sensor and the target move in straight lines after they choose their moving directions. More general mobility strategies would allow them to change direction and speed during their movements. However, we can see that such flexibility does not lead to a

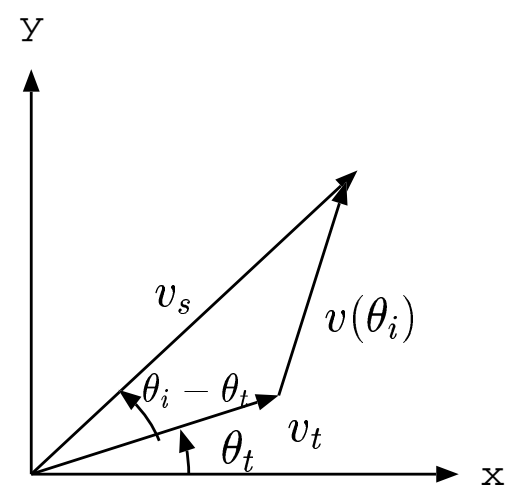

Figure 2: Relative sensor speed in the framework where target is stationary.

better result for either the sensor network or the target. If a sensor changes direction during its movement, there will be an overlap in its own covered area. This overlap causes the field to be covered less efficiently and will not help to detect the target sooner. Also, in order to speed up the search, each sensor should move at its maximum speed. Since the target only knows the mobility strategy of the sensors (sensor direction distribution density function) and it does not know the locations and directions of the sensors, changing direction and speed will not help the target to prolong its detection time.

To solve the minimax optimization problem, we first characterize the detection time of a target moving at a constant speed in a particular direction.

TheOREM 4. Consider a sensor network $B(\lambda, r)$ at time $t=0$, with sensors moving according to the random mobility model at a fixed speed $v_{s}$. Let $X$ be the detection time of a target moving at speed $v_{t}$ along direction $\theta_{t}$. Denote

$$
\begin{aligned}
& c=v_{t} / v_{s}, \quad \hat{c}=1+c \\
& w(u)=\sqrt{1-\frac{4 c}{\hat{c}^{2}} \cos ^{2} \frac{u}{2}} \\
& \bar{v}_{s}=v_{s} \hat{c} \int_{0}^{2 \pi} w\left(\theta-\theta_{t}\right) f_{\Theta}^{s}(\theta) d \theta
\end{aligned}
$$

We have

$$
X \sim \exp \left(2 \lambda r \bar{v}_{s}\right)
$$

Proof. Similar to the proof of Theorem 3, we divide the space evenly around point $p$ into $k$ directions $(k \rightarrow \infty)$. We now have $k$ sensor classes where class $i$ sensors move in the direction $\theta_{i}=\frac{2 \pi i}{k}$. Sensors of class $i$ form a Poisson point process with density $\lambda_{i}=\lambda f_{\Theta}^{s}\left(\theta_{i}\right) \Delta \theta$, where $\Delta \theta=2 \pi / k$.

Let $X_{i}$ be the time point $p$ is first reached by a sensor of class $i$; the detection time $X$ is the minimum of all of the first hit times, $X=\min X_{i}$.

Consider the reference framework where the target is stationary. We can compute the relative speed of class $i$ sensors to the target, $v\left(\theta_{i}\right)$, as illustrated in Figure 2. Let $c=v_{t} / v_{s}$ be the ratio of the target speed to the sensor speed. 
We have

$$
\begin{aligned}
v\left(\theta_{i}\right) & =\sqrt{\left(v_{s} \cos \left(\theta_{i}-\theta_{t}\right)-v_{t}\right)^{2}+v_{s}^{2} \sin ^{2}\left(\theta_{i}-\theta_{t}\right)} \\
& =\left(v_{s}+v_{t}\right) \sqrt{1-\frac{2 v_{s} v_{t}}{\left(v_{s}+v_{t}\right)^{2}}\left(1+\cos \left(\theta_{i}-\theta_{t}\right)\right)} \\
& =v_{s} \hat{c} \sqrt{1-\frac{4 c}{\hat{c}^{2}} \cos ^{2} \frac{\left(\theta_{i}-\theta_{t}\right)}{2}} \\
& =v_{s} \hat{c} w\left(\theta_{i}-\theta_{t}\right) .
\end{aligned}
$$

In the framework where class $i$ sensors are relatively stationary, point $p$ moves in direction $\left(2 \pi-\theta_{i}\right)$ at speed $v\left(\theta_{i}\right)$. From [13], the linear contact distance, $Y_{i}$, follows an exponential distribution with parameter $2 \lambda_{i} r$. Since $X_{i}=Y_{i} / v_{i}$, we have

$$
X_{i} \sim \exp \left(2 \lambda_{i} r v_{i}\right) .
$$

The minimum of these exponentially distributed first hit times is exponentially distributed with a parameter equal to the sum of the parameters of the individual exponential distributions:

$$
\begin{aligned}
\lim _{k \rightarrow \infty} \sum_{i=1}^{k} 2 \lambda_{i} r v_{i} & =\lim _{k \rightarrow \infty} \sum_{i=1}^{k} 2 \lambda f_{\Theta}^{s}\left(\theta_{i}\right) \Delta \theta v\left(\theta_{i}\right) r \\
& =2 \lambda r \int_{0}^{2 \pi} f_{\Theta}^{s}(\theta) v(\theta) d \theta \\
& =2 \lambda r \bar{v}_{s}
\end{aligned}
$$

where $\bar{v}_{s}=v_{s} \hat{c} \int_{0}^{2 \pi} w\left(\theta-\theta_{t}\right) f_{\Theta}^{s}(\theta) d \theta$.

In the following, we will refer to $\bar{v}_{s}$ as the effective sensor speed in the framework where the target is stationary.

From (6) and (10), it can be noted that the detection times of both moving targets and stationary targets follow exponential distributions, and that the parameters are of the same form, except the sensor speed in (6) is now replaced by the effective sensor speed in (10) for the mobile target case.

Assuming that the sensor density and sensing range are fixed, since the target detection time follows an exponential distribution of parameter $2 \lambda r \bar{v}_{s}$, maximizing the expected detection time corresponds to minimizing the effective sensor speed $\bar{v}_{s}$. In the following, we derive the optimal target mobility strategies for two special sensor mobility models.

- Sensors move in the same direction $\theta_{s}: f_{\Theta}^{s}(\theta)=\delta(\theta-$ $\left.\theta_{s}\right)$.

Using the fundamental property of the delta function $\int_{-\infty}^{\infty} f(x) \delta(x-a) d x=f(a)$, we have

$$
\begin{aligned}
\bar{v}_{s} & =v_{s} \hat{c} \int_{0}^{2 \pi} w\left(\theta-\theta_{t}\right) \delta\left(\theta-\theta_{s}\right) d \theta \\
& =v_{s} \hat{c} w\left(\theta_{s}-\theta_{t}\right)
\end{aligned}
$$

We need to choose a proper $\theta_{t}$ and $v_{t}$ that minimizes the above effective sensor speed $\bar{v}_{s}$. First, it is easy to see that we require $\theta_{t}=\theta_{s}$. Now, we have

$$
\bar{v}_{s}=v_{s} \hat{c} \sqrt{1-\frac{4 c}{\hat{c}^{2}}}=\left|v_{t}-v_{s}\right|
$$

and $\bar{v}_{s}$ is minimized when

$$
v_{t}= \begin{cases}v_{s} & \text { if } v_{t}^{\max } \geq v_{s} \\ v_{t}^{\max } & \text { otherwise. }\end{cases}
$$

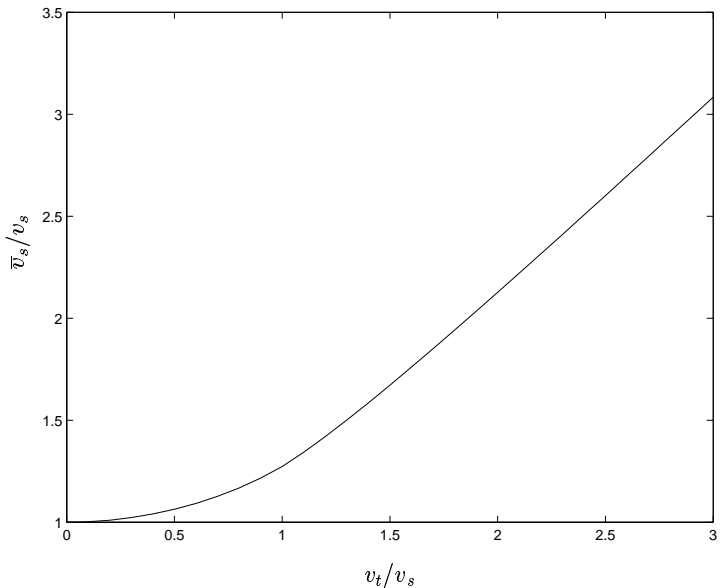

Figure 3: Normalized effective relative sensor speed $\bar{v}_{s} / v_{s}$ as a function of $c=v_{t} / v_{s}$

The above results show, quite intuitively, that the target should move in the same direction as the sensors at a speed closest matching the sensor speed. If the maximum target speed is greater than the sensor speed, the target will not be detected since it chooses to move at the same speed and in the same direction as the sensors. In this case, the detection time is infinity. Otherwise, if the maximum target speed is smaller than the sensor speed, the target should move at the maximum speed in the same direction of the sensors. The expected detection time is $\frac{1}{2 \lambda r\left(v_{s}-v_{t}^{\max }\right)}$.

- The direction of sensor movement is uniformly chosen within $[0,2 \pi): f_{\Theta}^{s}(\theta)=\frac{1}{2 \pi}$.

Figure 3 plots the normalized effective sensor speed $\bar{v}_{s} / v_{s}$ as a function of $c=v_{t} / v_{s}$, the ratio of the target speed to the sensor speed. The effective sensor speed is an increasing function of $c$, and is minimized when $c=0$, or $v_{t}=0$. Therefore, if each sensor uniformly chooses its moving direction from 0 to $2 \pi$, the maximum expected detection time is achieved when the target does not move. The corresponding expected detection time is $\frac{1}{2 \lambda r v_{s}}$. The optimal target mobility strategy in this case can be intuitively explained as follows. Since sensors move in all directions with equal probability, the movement of the target in any direction will result in a larger relative speed and thus a smaller first hit time in that particular direction. Consequently, the minimum of the first hit times in all directions (detection time) will become smaller.

We now present the solution to the minimax problem in the following theorem.

TheOREM 5. Consider a sensor network $B(\lambda, r)$ at time $t=0$, with sensors moving according to the random mobility model at a fixed speed $v_{s}$. The optimal mobility strategy of the sensors is for each sensor to choose a direction according to a uniform distribution, i.e., $f_{\Theta}^{s}(\theta)=\frac{1}{2 \pi}$.

Proof. For sensors, minimizing target detection time is equivalent to maximizing the effective sensor speed after a 
target selects the optimal speed and direction. We first prove for any given target speed $v_{t}$, that among all possible sensor direction distributions, the minimum effective sensor speed resulted from the optimal target direction choice, $\min _{\theta_{t}} \bar{v}_{s}$, is maximized when sensors choose directions according to a uniform distribution. The formal statement is described as follows.

Denote the uniform distribution density as $f_{\Theta}^{\text {uniform }}=$ $1 / 2 \pi$. From Theorem 4, the effective sensor speed is a function of sensor direction distribution density, target speed and direction, $\bar{v}_{s}\left(f_{\Theta}^{s}(\theta), \theta_{t}, v_{t}\right)=\int_{0}^{2 \pi} w\left(\theta-\theta_{t}\right) f_{\Theta}^{s}(\theta) d \theta$.

We will prove that

$$
\min _{\theta_{t}, v_{t}} \bar{\nu}_{s}\left(f_{\Theta}^{s}(\theta), \theta_{t}, v_{t}\right) \leq \min _{\theta_{t}, v_{t}} \bar{\nu}_{s}\left(f_{\Theta}^{\text {uniform }}, \theta_{t}, v_{t}\right)
$$

for all $f_{\Theta}^{s}(\theta)$.

First, let us consider the right-hand side of (11). We have

$$
\begin{aligned}
\bar{\nu}_{s}\left(f_{\Theta}^{\text {uniform }}, \theta_{t}, v_{t}\right) & =\frac{1}{2 \pi} \int_{0}^{2 \pi} w\left(\theta-\theta_{t}\right) d \theta \\
& =\frac{1}{2 \pi} \int_{-\theta_{t}}^{2 \pi-\theta_{t}} w(u) d u \\
& =\frac{1}{2 \pi} \int_{0}^{2 \pi} w(u) d u
\end{aligned}
$$

for all $\theta_{t}$, since the mapping $u \rightarrow w(u)$ is periodic with period $2 \pi$. This shows that

$$
\min _{\theta_{t}} \bar{\nu}_{s}\left(f_{\Theta}^{\text {uniform }}, \theta_{t}, v_{t}\right)=\frac{1}{2 \pi} \int_{0}^{2 \pi} w(u) d u .
$$

We now come back to the proof of (11). We have

$$
\begin{aligned}
& \min _{\theta_{t}} \bar{\nu}_{s}\left(f_{\Theta}^{s}(\theta), \theta_{t}, v_{t}\right) \\
\leq & \frac{1}{2 \pi} \int_{0}^{2 \pi} \bar{\nu}_{s}\left(f_{\Theta}^{s}(\theta), \theta_{t}, v_{t}\right) d \theta_{t} \\
= & \frac{1}{2 \pi} \int_{0}^{2 \pi} \int_{0}^{2 \pi} w\left(\theta-\theta_{t}\right) f_{\Theta}^{s}(\theta) d \theta d \theta_{t} \\
= & \frac{1}{2 \pi} \int_{0}^{2 \pi} f_{\Theta}^{s}(\theta)\left(\int_{\theta-2 \pi}^{\theta} w(u) d u\right) d \theta \\
= & \frac{1}{2 \pi}\left(\int_{0}^{2 \pi} f_{\Theta}^{s}(\theta) d \theta\right)\left(\int_{0}^{2 \pi} w(u) d u\right) \\
= & \frac{1}{2 \pi} \int_{0}^{2 \pi} w(u) d u \\
= & \min _{\theta_{t}} \bar{\nu}_{s}\left(f_{\Theta}^{\text {uniform }}, \theta_{t}, v_{t}\right)
\end{aligned}
$$

where the last three equalities follow from the fact that $w(u)$ is periodic with period $2 \pi$, from the fact that $f_{\Theta}^{s}(\theta)$ is a probabily density function on $[0,2 \pi]$, and from (12), respectively.

The proof of (11) is concluded by taking first the minimum over $v_{t}$ in the left-hand side of (13), then by taking the minimum over $v_{t}$ in the right-hand side of (13).

It follows from the proof of Theorem 5 that when sensors choose directions according to a uniform distribution, the optimal target mobility strategy is to stay stationary, $v_{t}=0$ (since $\bar{v}_{s}\left(f_{\Theta}^{\text {uniform }}, \theta_{t}, v_{t}\right)$ is maximized when $c=0$, i.e., when $\left.v_{t}=0\right)$, and $\theta_{t}$ is irrelevant in this case.

\section{RELATED WORK}

Recently, sensor deployment and coverage related topics have become an active research area. In this section, we present a brief overview of the previous work on the coverage of both stationary and mobile sensor networks that is most relevant to our study. A more thorough survey of the sensor network coverage is provided by [2].

Many previous studies have focused on characterizing coverage for stationary sensor networks. In [12], the authors considered a grid-based sensor network and derived the conditions for the sensing range and failure rate of sensors to ensure that an area is fully covered. In [8], the authors proposed several algorithms to find paths that are most or least likely to be detected by sensors in a sensor network. Path exposure of moving objects in sensor networks was formally defined and studied in [9], where the authors proposed an algorithm to find minimum exposure paths, along which the probability of a moving object being detected is minimized. The best coverage problem is further explored by Li et al in [6]. In [7], the authors defined several important coverage measures for a large-scale stationary sensor network, namely, the area coverage, detection coverage, and node coverage. Under the assumption that sensor location follows a Poisson point process, the authors obtained analytical results for the coverage measures under a Boolean sensing model and a general sensing model.

While the coverage of stationary sensor networks has been extensively studied and relatively well understood, researchers have started to explore the coverage of mobile sensor networks only recently. In [5], the authors proposed a potentialfield-based algorithm in which nodes are treated as virtual particles subjected to virtual force. The virtual forces repel the nodes from each other and from obstacles, and ensure that the initial configuration of nodes quickly spreads out to maximize coverage area. In [15], the authors presented another virtual-force-based sensor movement strategy to enhance network coverage after an initial random placement of sensors. The virtual force of a sensor is directly derived using the distance between the sensor and the other sensors and obstacles, and is used to compute the sensor movements that attempt to maximize the sensor field coverage. After the execution of the algorithm and once the effective sensor positions are identified, a one-time movement is carried out to redeploy the sensors. In [14], the authors proposed several algorithms that identify existing coverage holes in the network and compute the desired target positions where sensors should move in order to increase the coverage. All of these proposed algorithms strive to spread sensors in the field in order to obtain a stationary configuration such that the covered area is maximized. The main difference is how exactly the desired positions of sensors are computed.

Note that in this work, we study the coverage of a mobile sensor network from a very different perspective. Instead of trying to achieve an improved stationary network configuration as an end result of sensor movement, we have focused on the coverage capabilities resulted from the continuous movement of the sensors.

\section{SUMMARY}

In this paper, we study the dynamic aspects of the coverage of a mobile sensor network. More specifically, we characterized area coverage at specific time instants and during a 
time interval, and the detection time of a randomly located target. These coverage measures depend on the process of sensor movement and are unique attributes of mobile sensor networks.

For the random initial deployment strategy and the sensor mobility model under consideration, we have shown that while the area coverage at any given time instants remains unchanged, more area will be covered during a time interval, also, targets that will be detected in a stationary sensor network can now be detected by moving sensors. The tradeoff is that a location is only covered part of the time. This scenario is important for applications that do not require simultaneous coverage at specific time instants but rather desires to cover a large portion of the area during a certain time period.

We use detection time to measure how quickly sensors detect a randomly located target. The results suggests that sensor mobility can be exploited to effectively reduce the detection time of a stationary target when the number of sensors is limited. For mobile targets, the target detection time depends on sensor and target mobility strategies. We take a game theoretic approach and study the best worstcase performance of a mobile sensor network in terms of the target detection time. For a given sensor mobility behavior, we assume that a target can choose its mobility strategy so as to maximize its detection time, while sensors choose a mobility strategy that minimizes the maximum detection time. We have shown that the optimal sensor mobility strategy is that each sensor chooses its movement uniformly in all directions. The corresponding target mobility strategy is to stay stationary in order to maximize its detection time.

\section{REFERENCES}

[1] M. Batalin and G. Sukhatme. Spreading out: A local approach to multi-robot coverage. In 6th International Conference on Distributed Autonomous Robotic Systems (DSRS02), 2002.

[2] M. Cardei and J. Wu. Coverage in Wireless Sensor Networks in Handbook of Sensor Networks. CRC Press, 2004.

[3] T. Clouqueur, V. Phipatanasuphorn, P. Ramanathan, and K. K. Saluja. Sensor deployment stategy for target detection. In First ACM International Workshop on Wireless Sensor Networks and Applications, 2002.
[4] P. Hall. Introduction to the Theory of Coverage Processes. John Wiley \& Sons, 1988.

[5] A. Howard, M. Mataric, and G. Sukhatme. Mobile sensor network deployment using potential fields: A distributed, scalable solution to the area coverage problem. In DARS 02, 2002.

[6] X.-Y. Li, P.-J. Wan, and O. Frieder. Coverage problems in wireless ad-hoc sensor networks. IEEE Transactions on Computers, 52(6):753-763, June 2003.

[7] B. Liu and D. Towsley. A study on the coverage of large-scale sensor networks. In The 1st IEEE International Conference on Mobile Ad-hoc and Sensor Systems, 2004.

[8] S. Meguerdichian, F. Koushanfar, M. Potkonjak, and M. B. Srivastava. Coverage problems in wireless ad-hoc sensor networks. In Proc. IEEE Infocom, pages 1380-1387, 2001.

[9] S. Meguerdichian, F. Koushanfar, G. Qu, and M. Potkonjak. Exposure in wireless ad-hoc sensor networks. In Mobile Computing and Networking, pages 139-150, 2001.

[10] J. Pearce, P. Rybski, S. Stoeter, and N. Papanikolopoulous. Dispersion behaviors for a team of multiple miniature robots. In IEEE International Conference on Robotics and Automation, 2003.

[11] R. Serfozo. Introduction to Stochastic Networks. Springer, 1999.

[12] S. Shakkottai, R. Srikant, and N. Shroff. Unreliable sensor grids: Coverage, connectivity and diameter. In Proc. IEEE Infocom, 2003.

[13] D. Stoyan, W. S. Kendall, and J. Mecke. Stochastic Geometry and its Applications: Second Edition. John Wiley and Sons, 1995.

[14] G. Wang, G. Cao, and T. L. Porta. Movement-assisted sensor deployment. In Proc. IEEE Infocom, 2004.

[15] Y. Zou and K. Chakrabarty. Sensor deployment and target localization based on virtual forces. In Proc. IEEE Infocom, 2003. 